\title{
Adaptive Sampling Rate Assignment for Block Compressed Sensing of Images Using Wavelet Transform
}

\author{
Luo Xin ${ }^{1}$, Zhang Junguo ${ }^{1, *}$, Chen Chen $^{2}$ and Lin Fantao ${ }^{3}$ \\ ${ }^{I}$ School of Technology, Beijing Forestry University, Beijing 100083, China; ${ }^{2}$ Department of Electrical Engineering, \\ University of Texas at Dallas, Richardson 75080, USA, ${ }^{3}$ China Electric Power Research Institute, Beijing, 100192, China
}

\begin{abstract}
Compressed sensing theory breaks through the limit of two times the bandwidth of the signal sampling rate in Nyquist theorem, providing a guideline for new methods of image acquisition and compression. For still images, block compressed sensing (BCS) has been designed to reduce the size of sensing matrix and the complexity of sampling and reconstruction. However, BCS algorithm assigns the same sampling rate for all the image blocks without considering the structures of the blocks. In this paper, an adaptive sampling rate assignment method is presented for BCS of images using wavelet transform. Wavelet coefficients of an image can reflect the structure information. Therefore, adaptive sampling rates were calculated and assigned to the image blocks based on their wavelet coefficients. Several standard test images were employed to evaluate the performance of the proposed algorithm. Experimental results demonstrate that the proposed algorithm provided superior performance on both the reconstructed image quality and the visual effect.
\end{abstract}

Keywords: Adaptive sampling rate assignment, Block compressed sensing, Still image, Wavelet transform.

\section{INTRODUCTION}

With the rapid development of information technology, the demand for image information is growing. Meanwhile, how to store, process and transmit large amounts of image data have become a serious problem. Traditional still image compression standard $[1,2]$ : JPEG and JPEG2000 can remove image's spatial redundancy and obtain a large compression ratio. However, there are some drawbacks: (1) Based on the Nyquist sampling theorem, the sampling rate cannot be less than two times of the signal bandwidth. It may be difficult for a hardware system to meet a high sampling rate. (2) All the image transform coefficients are calculated, but only a few are retained. This leads to a waste of data computing and memory resources. (3) During data transmission, missing coefficients will affect the image reconstruction quality.

In 2006, E. Candes, D. Donoho and T. Tao proposed the Compressed Sensing (CS) theory [3-5]. Under certain conditions, a signal can be recovered from the distance in fewer samples than required by the Nyquist sampling theorem. CS theory provides a breakthrough for image compression. However, for two-dimensional images, CS algorithm faces high computational complexity and large memory access. In order to solve these problems, Lu Gan proposed block-based compressed sensing (BCS) [6]. In the BCS, an image is divided into blocks and sampling and reconstruction are conducted in a block-by-block manner. Therefore, the computational complexity of sampling and reconstruction are greatly reduced.

*Address correspondence to this author at School of Technology, Beijing Forestry University, Qinghua East Road, Haidian District, Beijing, 100083, P.R. China; Tel: 18810381696; E-mail: zhangjunguo@bjfu.edu.cn
The BCS algorithm assigns the same sampling rate for all blocks within an image. However, due to piecewise smooth regions in an image, some image blocks may contain only a little information. For example, the texture blocks contain more information (e.g., edges) than the smooth blocks. Blocks with little information can be reconstructed by fewer observations. Therefore, it is not necessary to assign the same sampling rate for all image blocks. The image structural information can be utilized, under the premise of total sampling rate unchanged, to adaptively allocate sampling rate for different image blocks.

Wavelet transform is an efficient image representation method that has been extensively used in image compression. It can capture the edge information in different scales and orientations. In our proposed adaptive sampling rate assignment method, wavelet transform coefficients of an image are used to analyze the structural information of each image block and adaptively allocate sampling rate for each block. The proposed adaptive sampling rate assignment method for BCS can improve the reconstruction performance significantly. The rest of this paper is organized as follows. Section 2 gives a brief review of CS and BCS. Section 3 describes the proposed adaptive CS algorithm of still images based on wavelet coefficients. Experimental results are given in Section 4. Finally, Section5 is the conclusion.

\section{COMPRESSED SENSING}

\subsection{The Basic Principles of Compressed Sensing}

A real-valued signal $x \in R^{N}$ is considered, which is sparse with respect to a transform - Then $x=\Psi \alpha$ and $\|\alpha\|_{0}=K \ll N$ (Where $\|\alpha\|_{0}$ represents the number of 
non-zero coefficient of vector $\alpha$ ); the coefficient vector $\alpha$ can be reconstructed with high probability from its linear and non-relevant projections $\mathrm{y}$.

$$
y=\Phi x=A \alpha
$$

Here, $y \in R^{M}$ is the vector of projections constituted by a small amount of linear, non-relevant measured values. $\Phi \in R^{M \times N}$ is a measurement matrix and $M=N . A \in R^{M \times N}$ is on behalf of $\Phi \Psi$, called the sensing matrix. Under normal circumstances, $K<M=N$.

In essence, $y$ can be regarded as a linear combination of all the columns of A corresponding $\alpha_{i} \neq 0$. Formula (2-1) actually implements a dimensionality reduction process and can also be considered a data compression method. Compared to the dimensionality reduction process, we are more concerned about how to recover $\alpha$ from the coefficient vector $y$. That is solving the following equation:

$\min _{\alpha}\|\alpha\|_{0}$, s.t. $\mathrm{y}=\mathrm{A} \alpha$

Since Eq. (2-1) is a group of underdetermined linear equations, solving (2-2) is NP-hard. But in the case of $\alpha$ being sparse, if $A$ satisfies the restricted isometry property (RIP) [5], then the problem can be solved by iterative greedy algorithms, such as OMP [7], StOMP [8] and CoSaMP [9]. After obtaining $\alpha$, the original $x$ can be reconstructed by $x=\Psi \alpha$.

\subsection{Block Compressed Sensing}

When CS is used to process image signal, the size of measurement matrix $\Phi \in R^{M \times N}$ will be very large, while $N$ is typically between $10^{4}$ and $10^{6}$, making the storage and computing very challenging. In order to solve this problem, Lu Gan proposed a compressed sensing method based on a block-based sampling strategy [6].

BCS procedure is described as follows. By considering an image with $N=I_{r} \times I_{c}$ pixels in total, the image is divided into small non-overlapping blocks, each with the size of $B \times B$. Let $x_{m}$ represents the vectorized signal of the $\mathrm{m}$-th block, $m=1,2 \mathrm{~L} n, n=N / B^{2}$, then each block is sampled with the same measurement matrix $\Phi_{B}$. The corresponding output CS vector $y_{m}$ can be written as

$y_{m}=\Phi_{B} x_{m}$

Where $\Phi_{B}$ is a matrix with the size of $M_{B} \times B^{2}$, and $M_{B}=\left\lfloor\right.$ subrate $\left.\times B^{2}\right\rfloor, 0 \leq$ subrate $\leq 1$. For the whole image, the equivalent sampling operator $\Phi$ in (2-1) is thus, a block diagonal matrix of the following form:
$\Phi=\left(\begin{array}{llll}\Phi_{B} & 0 & \mathrm{~L} & 0 \\ 0 & \Phi_{B} & & \mathrm{M} \\ \mathrm{M} & & 0 & 0 \\ 0 & \mathrm{~L} & 0 & \Phi_{B}\end{array}\right)$.

Compared with the compressed sensing based on the whole image, BCS is faster; however, it poses serious blocking artifacts. To remove the blocking artifacts, BCS-SPL [10] introduces Wiener filtering into the image reconstruction process, and gradually improves the quality of reconstruction by iterative process. A BCS-SPL variant named MH-BCS-SPL was introduced [11] to further improve the BCS-SPL using a post-processing procedure where multihypothesis prediction strategy was used. The multihypothesis prediction strategy was also employed for hyperspectral image reconstruction [12] and single-image super-resolution [13]. Given the computational efficiency and good reconstruction performance of BCS-SPL, this paper adopted the BCS-SPL as the CS reconstruction method to investigate the adaptive sampling rate assignment for the BCS-SPL.

\section{ADAPTIVE COMPRESSED SENSING ALGO- RITHM}

\subsection{Overall Framework of Adaptive Compressed Sensing Method}

Different from traditional imaging systems, CS belongs to the sampling process information, and each CS measurement contains global information of the image block. The measurements contain useful information making a significant impact on the reconstruction result. The original BCS algorithm assigns the same sampling rate for all the image blocks. However, when an image is divided into small blocks, the different image blocks contain different amount of information. Blocks containing little information can be reconstructed by fewer observations than those containing more information. Thus it is possible to adaptively allocate different sampling rates for different blocks to improve the BCS-SPL reconstruction performance.

The framework of the proposed adaptive compressed sensing algorithm for images is presented in Fig. (1). It includes adaptive sampling rate assignment, CS sampling, quantization, coding and CS reconstruction. This paper focused on the part of adaptive sampling assignment for different blocks.

\subsection{Adaptive Sampling Rate Assignment using Wavelet Transform}

Wavelet analysis is a time - frequency analysis method of signal. It has the characteristics of multiscale analysis. In both the domains of time and frequency, wavelet analysis has the ability to characterize local signal characteristics. It has a high frequency resolution and a low time resolution in the low frequency domain and a low frequency resolution and a high time resolution in the high frequency domain. It is particularly suitable for analyzing and detecting transient abnormality signal in the normal signal.

After wavelet transform, the transform coefficients of an image can be categorized into low frequency coefficients and 


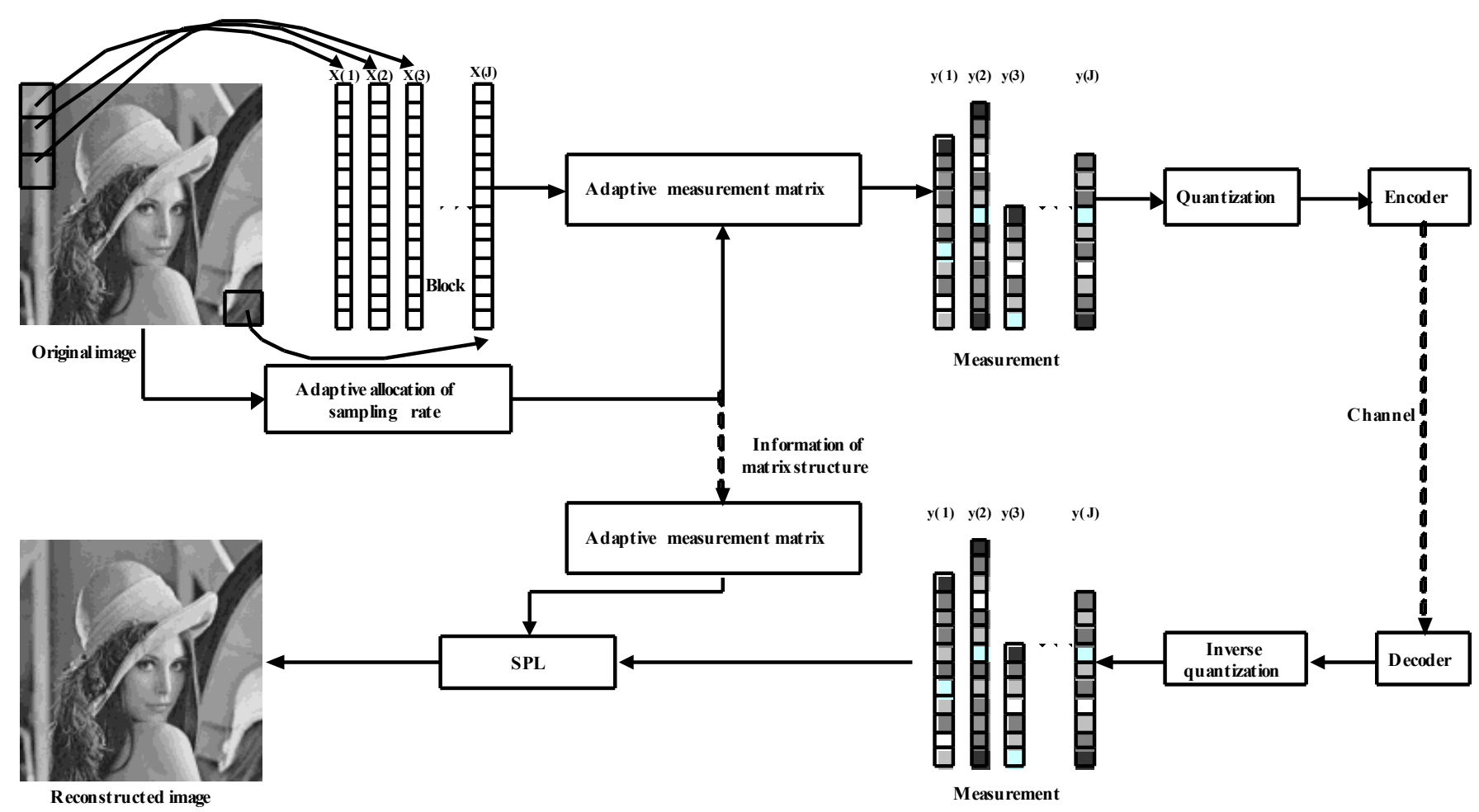

Fig. (1). Overall framework of the proposed adaptive compressed sensing method.

high frequency coefficients. In fact, the low frequency coefficients and high frequency coefficients are two different types of signals. Low-frequency coefficients epitomize the image energy information, while, the high-frequency coefficients save rich detailed information of an image. Therefore, an image can be observed and analyzed by its wavelet coefficients, as shown in Fig. (2).

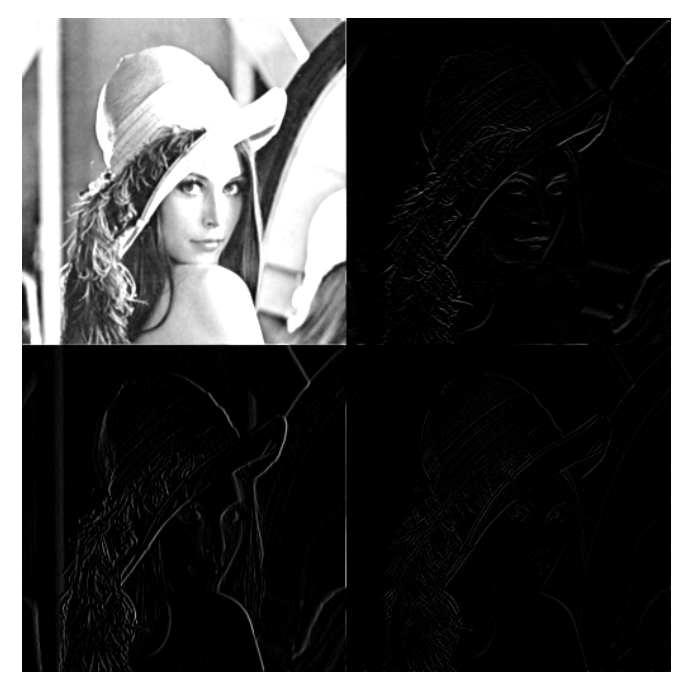

Fig. (2). The discrete stationary wavelet transform of the Lena image.

This paper presents an adaptive compressed sensing algorithm of still images based on wavelet coefficients. For easy operation, the paper used a discrete stationary wavelet transform (SWT). Therefore, the proposed algorithm is named as
SWT-BCS-SPL. Compared with the conventional discrete wavelet transform (DWT), SWT has the "translation invariance" property [14]. The number of wavelet coefficients of the sub-band layers after decomposition is equal to the number of original image pixels, therefore, the SWT was applied on the whole image, after which the image was divided into blocks in the transform domain. Finally, the statistical results were calculated as the absolute values of the vertical and diagonal high-frequency coefficients of each block. The sampling rate allocation was determined according to the absolute value of high-frequency coefficients of each block. The detailed process is shown in Fig. (3).

The value of the sampling rate $S R$ was known, the size of the block was $B \times B$, the number of image blocks was $n$. Thus, the total number of measurements was obtained as $M=S R \times B^{2} \times n$. In this paper, the sampling upper bound is denoted as upper $=0.9 \times B^{2}$. In order to guarantee the basic quality of the reconstructed image, each block was assigned with the same fixed base sampling rate $F S R=W \times S R$, where $W$ is a parameter that decides a fixed sampling rate. If $W$ is larger, the fixed sampling rate for the blocks is higher, and $0 \leq W \leq 1$. According to the fixed sampling rate $F S R$, the fixed measurement number of each image block can be determined by $F M_{i}=\frac{F S R \times M}{n}$. After applying discrete stationary wavelet transform on the original image, the coefficient image was divided into blocks. The statistical results of the absolute values of the high-frequency coefficients of each block, denoted as $\operatorname{coef}\left(x_{i}\right)$, were calculated and the 

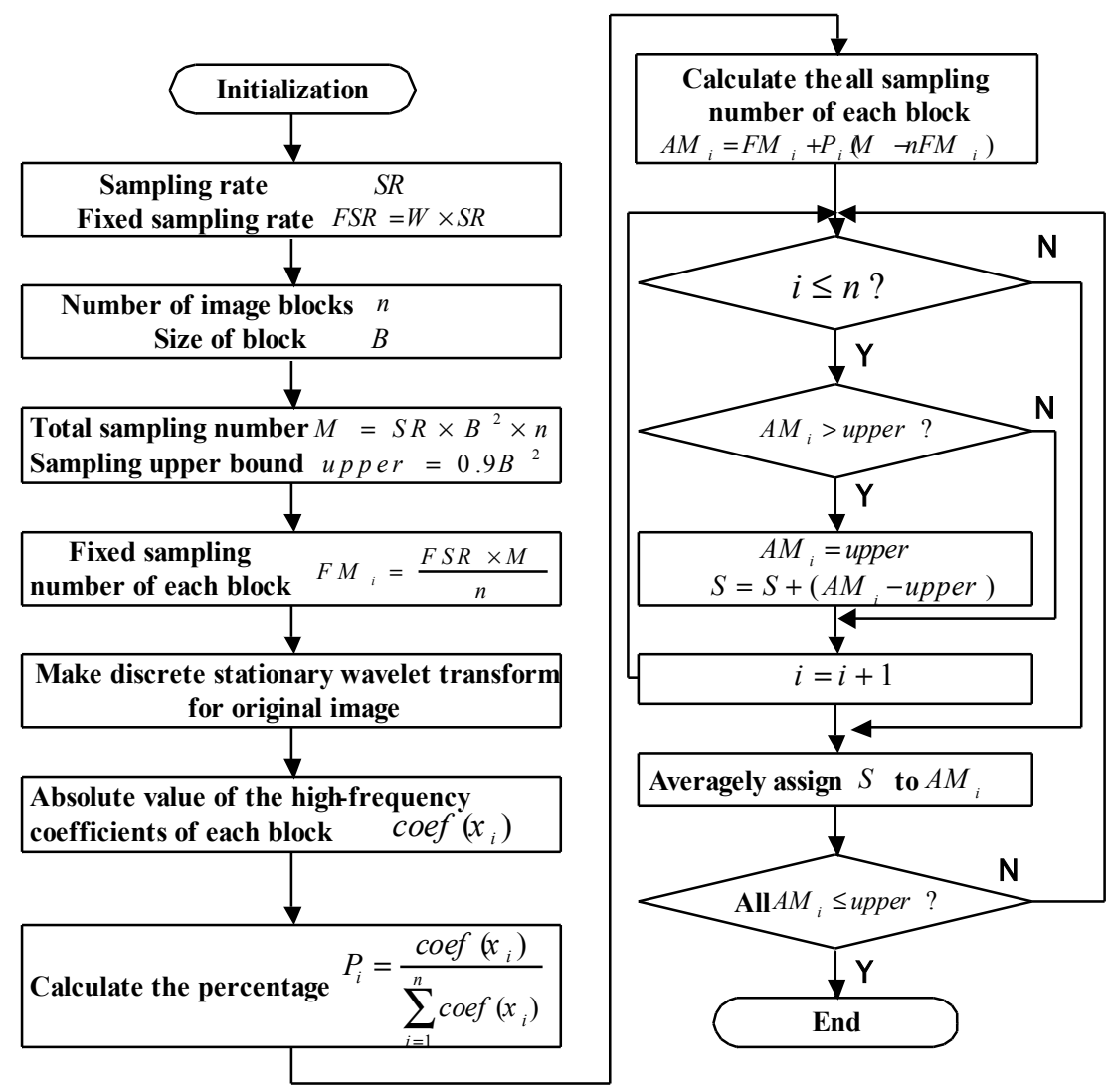

Fig. (3). Flow chart of the sampling rate assignment strategy based on wavelet coefficients.

percentage of $\operatorname{coef}\left(x_{i}\right)$ with respect to the whole image was obtained by $P_{i}=\frac{\operatorname{coef}\left(x_{i}\right)}{\sum_{i=1}^{n} \operatorname{coef}\left(x_{i}\right)}$.

The study computed the total number of measurements for each block according to $A M_{i}=F M_{i}+P_{i}\left(M-n F M_{i}\right)$. If the total number of measurements for a block is larger than the upper bound, the excess measurements are accumulated for each block to obtain the total excess measurements for the whole image, $S=S+\left(A M_{i}\right.$-upper $)$. The excess measurements were distributed equally to the blocks with $A M_{i}<$ upper. This process can be repeated until all the blocks with sampling measurements are within the range of the sampling upper bound. Here, the final number of measurements for each block is denoted as $A M_{i}$.

According to the sampling number of each block $A M_{i}$, the corresponding random measurement matrix was constructed to carry out CS measurement. Since the decoder was needed to reconstruct the measurement matrix, the information of matrix structure was also needed to be transmitted. Compared with the original BCS, the adaptive compressed sensing algorithm transmitted the sampling number of each block to the decoder as well. Therefore, the bits cost increased.

\section{EXPERIMENTAL RESULTS}

In this section, the performance of the proposed adaptive compressed sensing algorithm based on wavelet coefficients (SWT-BCS-SPL) was evaluated to compare it with the original BCS-SPL algorithm. For a more comprehensive evaluation of the reconstructed image quality, in addition to the commonly used PSNR, this paper used structural similarity index measurement system (SSIM) [15] as an additional image quality evaluation criterion.

Six test images were used in the experiments including Lena, Barbara, Goldhill, Barbara2, Boat and Cameraman, as shown in Fig. (4). All the images had the size of $512 \times 512$ pixels. The experiments were conducted using MATLAB v7.8 (R2009a) on a PC with an Intel(R) Core(TM)2 Duo CPU T6670 of $2.19 \mathrm{GHz}$ and 2-GB of RAM. The sparse transform in the BCS-SPL is the wavelet transform. It should be noted that all reconstruction evaluations (PSNR and SSIM) were averaged over ten independent trials, since the performance of reconstruction varied due to randomness of the sampling matrix.

\subsection{Parameter Tuning}

In order to analyze the effect of the two main parameters in the adaptive sampling algorithm, block size B and the fixed sampling rate allocation parameter $\mathrm{W}$, Lena image was selected as the test image for parameter tuning experiments. 


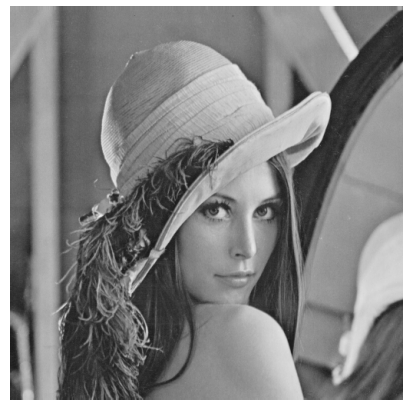

Lena

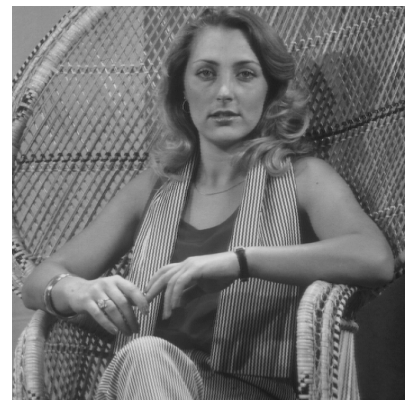

Barbara 2

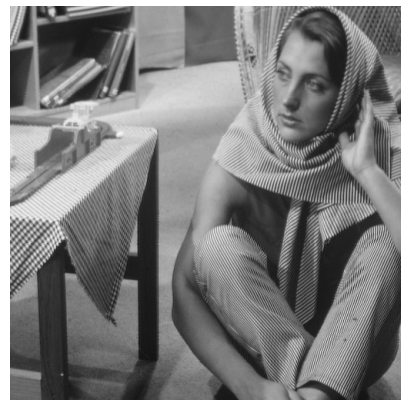

Barbara

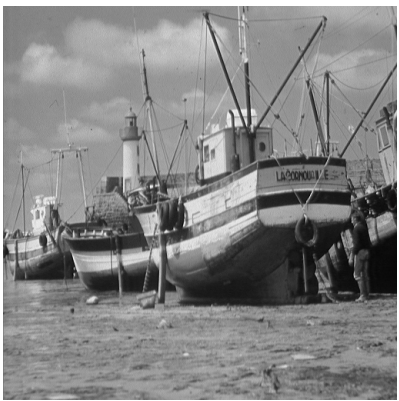

Boat

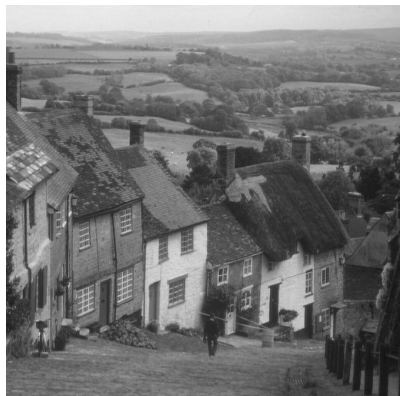

Goldhill

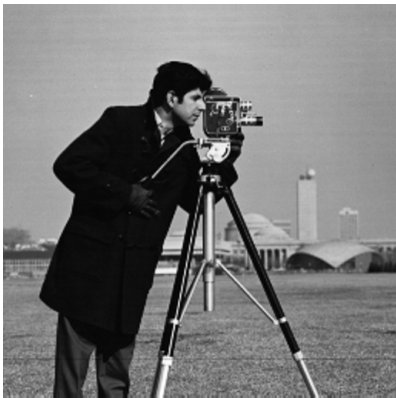

Cameraman

Fig. (4). Test images in our experiments.

Table 1. The relation between the PSNR of the reconstructed image and the block size.

\begin{tabular}{|c|c|c|c|c|}
\hline \multirow{2}{*}{ Algorithm } & $\mathbf{4} \times \mathbf{4}$ & $\mathbf{8} \times \mathbf{8}$ & $\mathbf{1 6 \times 1 6}$ & $\mathbf{3 2 \times 3 2}$ \\
\cline { 2 - 5 } & $\mathbf{P S N R}$ & $\mathbf{P S N R}$ & $\mathbf{P S N R}$ & $\mathbf{P S N R}$ \\
\hline \hline SWT-BCS-SPL & 38.15 & 38.73 & 38.48 & 38.26 \\
\hline
\end{tabular}

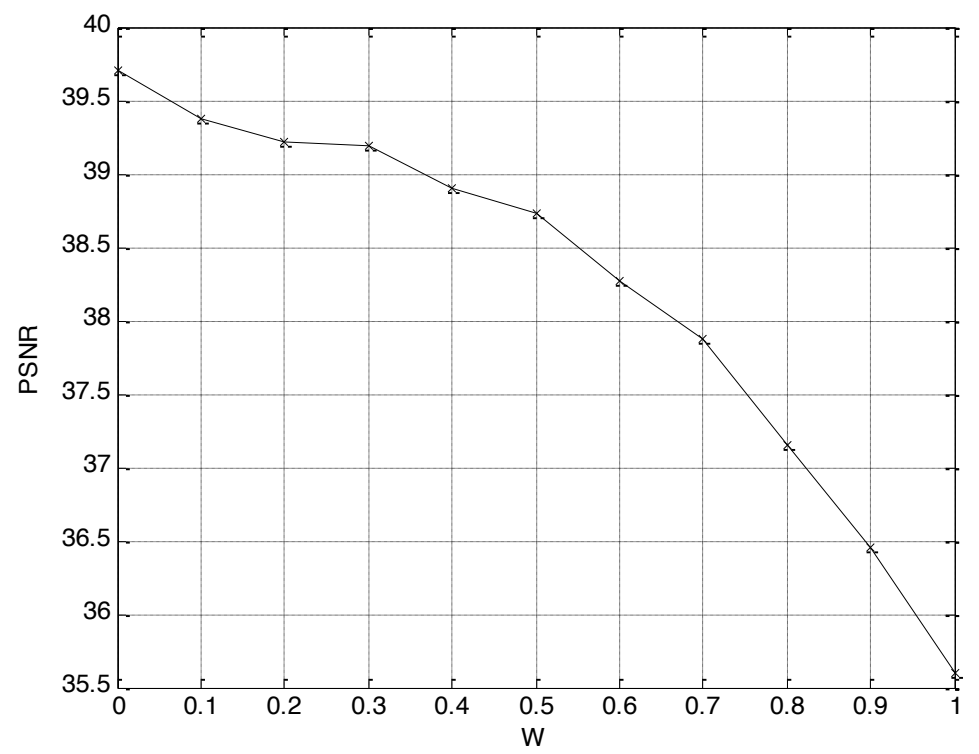

Fig. (5). The relation between PSNR of the reconstructed image and parameter $W$.

Under the experimental condition, when the sampling rate was 0.5 and the fixed sampling rate allocation parameter $\mathrm{W}$ was 0.5 , Table 1 shows the relation between the PSNR of the reconstructed image and the block size. It can be observed that when the block size was $8 \times 8$, the image reconstructed by SWT-BCS-SPL algorithm achieved the highest
PSNR value. Therefore, in the experiments, the block size B was set to $8 \times 8$.

Next, the paper analyzed the parameter $W$. The sampling rate was set to 0.5 and the block size B to $8 \times 8$, and a set of $W$ was tested. Fig. (5) shows the relation between the PSNR of the reconstructed image and the fixed sampling rate 
Table 2. Performance comparison between BCS-SPL and SWT-BCS-SPL at different sampling rates.

\begin{tabular}{|c|c|c|c|c|c|c|c|c|c|c|}
\hline \multirow[b]{3}{*}{ Algorithm } & \multicolumn{10}{|c|}{ Sampling Rate } \\
\hline & \multicolumn{2}{|c|}{0.1} & \multicolumn{2}{|c|}{0.2} & \multicolumn{2}{|c|}{0.3} & \multicolumn{2}{|c|}{0.4} & \multicolumn{2}{|c|}{0.5} \\
\hline & PSNR & SSIM & PSNR & SSIM & PSNR & SSIM & PSNR & SSIM & PSNR & SSIM \\
\hline & \multicolumn{10}{|c|}{ Lena } \\
\hline BCS-SPL & 26.37 & 0.79 & 30.02 & 0.87 & 32.16 & 0.90 & 34.10 & 0.93 & 35.65 & 0.94 \\
\hline \multirow[t]{2}{*}{ SWT-BCS-SPL } & 25.78 & 0.79 & 31.29 & 0.88 & 34.29 & 0.91 & 36.83 & 0.94 & 38.73 & 0.95 \\
\hline & \multicolumn{10}{|c|}{ Barbara } \\
\hline BCS-SPL & 22.04 & 0.65 & 23.65 & 0.74 & 24.77 & 0.80 & 26.15 & 0.85 & 27.51 & 0.89 \\
\hline \multirow[t]{2}{*}{ SWT-BCS-SPL } & 20.90 & 0.63 & 24.29 & 0.76 & 27.21 & 0.84 & 30.55 & 0.90 & 32.67 & 0.92 \\
\hline & \multicolumn{10}{|c|}{ Goldhill } \\
\hline BCS-SPL & 26.06 & 0.67 & 28.49 & 0.77 & 30.07 & 0.83 & 31.47 & 0.87 & 32.73 & 0.90 \\
\hline \multirow[t]{2}{*}{ SWT-BCS-SPL } & 23.69 & 0.66 & 28.63 & 0.78 & 30.36 & 0.83 & 32.42 & 0.87 & 33.98 & 0.90 \\
\hline & \multicolumn{10}{|c|}{ Barbara2 } \\
\hline BCS-SPL & 23.21 & 0.63 & 25.29 & 0.76 & 26.94 & 0.83 & 28.67 & 0.88 & 30.26 & 0.91 \\
\hline \multirow[t]{2}{*}{ SWT-BCS-SPL } & 22.86 & 0.64 & 26.18 & 0.78 & 28.94 & 0.85 & 31.48 & 0.90 & 33.33 & 0.93 \\
\hline & \multicolumn{10}{|c|}{ Boat } \\
\hline BCS-SPL & 23.97 & 0.66 & 27.11 & 0.77 & 29.07 & 0.83 & 30.81 & 0.87 & 32.25 & 0.90 \\
\hline \multirow[t]{2}{*}{ SWT-BCS-SPL } & 23.76 & 0.67 & 28.25 & 0.79 & 30.63 & 0.84 & 32.69 & 0.88 & 34.07 & 0.90 \\
\hline & \multicolumn{10}{|c|}{ Cameraman } \\
\hline BCS-SPL & 24.08 & 0.81 & 28.01 & 0.90 & 30.60 & 0.94 & 33.11 & 0.96 & 34.89 & 0.97 \\
\hline SWT-BCS-SPL & 24.65 & 0.83 & 30.57 & 0.92 & 34.76 & 0.95 & 39.13 & 0.97 & 42.13 & 0.98 \\
\hline
\end{tabular}
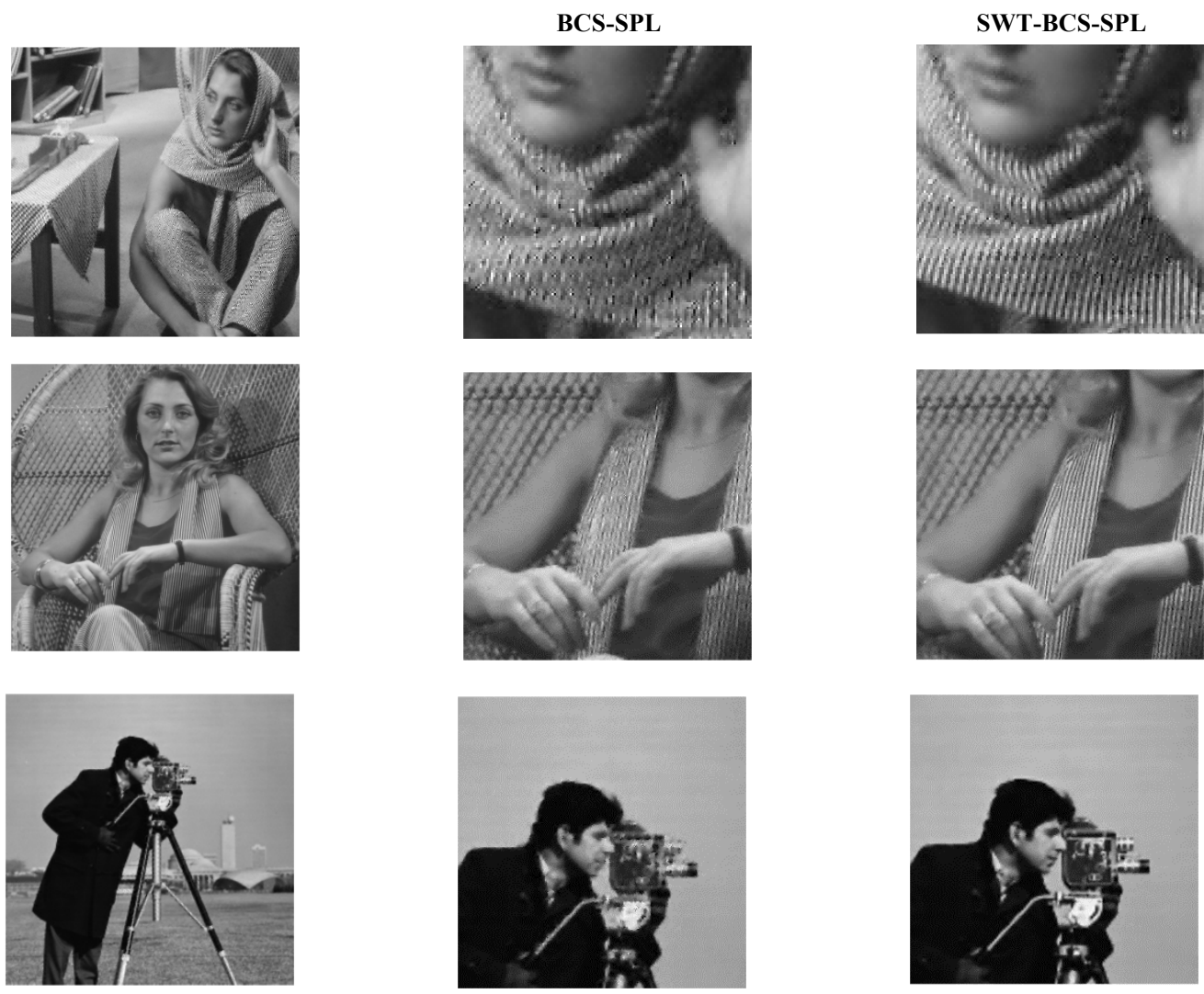

Fig. (6). Visual comparison of three reconstructed $512 \times 512$ images (shown in detail) for a sampling rate of $\mathrm{SR}=0.3$. 
allocation parameter $W$. It is obvious from the figure that, with the fixed sampling rate, allocation parameter increased, and the PSNR of the reconstructed image by SWT-BCS-SPL algorithm decreased. This also indicates that the adaptive sampling rate allocation can improve the quality of the reconstructed image. In this paper, the parameter $W$ was set to an intermediate value of 0.5 .

\subsection{Comparison with BCS-SPL}

The performance (PSNR and SSIM) of BCS-SPL and the proposed SWT-BCS-SPL at different sampling rates is shown in Table 2 . In the table, the best performance is emphasized by bold-faced font. It can be observed that SWTBCS-SPL showed improvements in almost all the sampling rates. These comparisons highlight the role of adaptive sensing. The results demonstrate that the proposed algorithm can improve the quality of the reconstructed image. The results show that the improvement in the texture detail-rich images (e.g. Barbara and Barbara2) was more obvious. For Barbara image, the maximal PSNR gain reached $5.16 \mathrm{~dB}$ and the average one was $2.3 \mathrm{~dB}$. For Barbara 2 image, the maximal PSNR gain was $3.07 \mathrm{~dB}$ and the average one was $1.68 \mathrm{~dB}$. When the total sampling rate was rather low (e.g. $\mathrm{SR}=0.1$, the advantage of SWT-BCS-SPL was not very clear and it performed worse than the BCS-SPL. The reason is that, since the total sampling number was very small, after adaptive allocation, the sampling number of each block was similar, therefore, SWT-BCS-SPL was not effective.

To better illustrate the improvement, a visual comparison is provided in Fig. (6). From the figure, it can be seen that the details of three images recovered with SWT-BCS-SPL are more clear than BCS-SPL. It is because in SWT-BCSSPL, relatively high sampling rates were assigned to the edge and rich texture blocks and low sampling rates were assigned to the smooth blocks.

\section{CONCLUSION}

In this paper, an adaptive compressed sensing algorithm of still images based on wavelet coefficients was proposed to improve the reconstruction performance of the block-based compressed sensing (BCS). The algorithm utilized wavelet coefficients of each image block as a sampling rate allocation criteria to adaptively assign sampling rates for each block. High sampling rates were assigned to the blocks containing detailed information (e.g., edges) and low sampling rates were assigned to the blocks containing less information (e.g., smooth background). Compared with the original BCS with uniform sampling rate for all the blocks, the experimental results demonstrated a significant improvement in the numerical and geometrical accuracy over the traditional block compressed sensing. However, since wavelet transform is complex, the computational complexity of the algorithm increased. In our future work, a computationally efficient sampling rate assignment strategy will be investigated.

\section{CONFLICT OF INTEREST}

The authors confirm that this article content has no conflict of interest.

\section{ACKNOWLEDGEMENTS}

This work was funded by Beijing Higher Education Young Elite Teacher Project (Grant No.YETP0760), Fundamental Research Funds for the Central Universities (Grant No.TD2013-3) and Import Project under China State Forestry Administration (Grant No.2014-4-05).

\section{REFERENCES}

[1] G.K. Wallace, "The JPEG still picture compression standard", Communications of the ACM, vol. 34, pp. 30-44, 1991.

[2] D.S. Taubman, and M.W. Marcellin, "JPEG2000: Standard for interactive imaging", In: Proceedings of the IEEE, vol. 90, pp. 1336-1357, 2002.

[3] D. L. Donoho, "Compressed sensing", IEEE Transactions on Information Theory, vol. 52, pp. 1289-1306, 2006.

[4] E. J. Candès, "Compressive sampling", In: Proceedings of the International Congress of Mathematicians, Switzerland, 2006, pp. 1433-1452.

[5] E. J. Candès, J. Romberg and T Tao, "Robust uncertainty principles: Exact signal reconstruction from highly incomplete frequency information", IEEE Transactions on Information Theory, vol. 52, pp. 489-509, 2006.

[6] L. Gan, "Block compressed sensing of natural images", In: Proceedings of the International Congress on Digital Signal Processing, UK, 2007, pp. 403-406.

[7] J. A. Tropp, and A. C. Gilbert, "Signal recovery from random measurements via orthogonal matching pursuit", IEEE Transactions on Information Theory, vol. 53, pp. 4655-4666, 2007.

[8] D. L. Donoho, Y. Tsaig and J. L. Starck, "Sparse solution of underdetermined systems of linear equations by stagewise orthogonal matching pursuit", IEEE Transactions on Information Theory, vol. 58, pp. 1094-1121, 2012.

[9] D. Needell, and J.A. Tropp, "CoSaMP: Iterative signal recovery from incomplete and inaccurate samples", Applied and Computational Harmonic Analysis, vol. 26, pp. 301-321, 2009.

[10] S. Mun, and J. E. Fowler, "Block compressed sensing of images using directional transforms", In: Proceedings of the International Congress on Image Processing, Cairo, Egypt, 2009, pp. 3021-3024.

[11] C. Chen, E. W. Tramel, and J. E. Fowler, "Compressed-sensing recovery of images and video using multihypothesis predictions," In: Proceedings of the 45th Asilomar Conference on Signals, Systems, and Computers, Pacific Grove, CA, 2011, pp. 1193-1198.

[12] C. Chen, W. Li, E. W. Tramel, and J. E. Fowler, "Reconstruction of hyperspectral imagery from random projections using multihypothesis prediction," IEEE Transactions on Geoscience and Remote Sensing, vol. 52, pp. 365-374, 2014.

[13] C. Chen, and J. E. Fowler, "Single-Image Super-Resolution Using Multihypothesis Prediction," In: Proceedings of the $46^{\text {th }}$ Asilomar Conference on Signals, Systems, and Computers, Pacific Grove, CA, pp. 608-612, 2012.

[14] G. Anbarjafari, S. Izadpanahi, and H. Demirel, "Video resolution enhancement by using discrete and stationary wavelet transforms with illumination compensation", Signal, Image and Video Processing, vol. 9, no. 1, pp. 1-6, 2012.

[15] Z. Wang, A. C. Bovik, and H. R. Sheikh, "Image quality assessment: from error visibility to structural similarity", IEEE Transactions on Image Processing, vol. 13, pp. 600-612, 2004. 